\title{
Evaluación del crecimiento de la población y transformación del uso de suelo urbano en la Zona Metropolitana de Pachuca, México
}

\section{Evaluation of population growth and the transformation of urban land use in the metropolitan area of Pachuca, Mexico}

\author{
Marcelino García-Benítez* \\ Laura Myriam Franco-Sánchez** \\ José Aurelio Granados-Alcantar**
}

Recibido: abril 09 de 2019

Aceptado: septiembre 04 de 2019

Resumen

Esta investigación identifica los procesos de crecimiento generados por el incremento de la población en las localidades rural-urbanas que han incidido en la transformación del suelo actual en distintas etapas del tiempo en los municipios que integran la Zona Metropolitana de Pachuca (ZMP). Además, evalúa el crecimiento de las localidades por el volumen de población mediante un análisis espacial, donde son utilizadas las proyecciones de la población total por localidad en el periodo 2010-2030, así como los procesos que han originado la susceptibilidad del suelo natural para ser transformado en urbano propiciando la extensión de la mancha urbana. Los resultados se obtienen mediante la aplicación del método de interpolación por isolíneas en el SIG (Sistemas de Información Geográfica), en el cual se establece la distribución espacial de la población según la tipología de las localidades.

Palabras clave: crecimiento urbano y uso de suelo.

\begin{abstract}
This research identifies the growth processes generated by the increase of the population in rural-urban localities, which have influenced the transformation of current land at different stages of time in the municipalities that integrate the Pachuca Metropolitan Area (ZMP). In addition, it evaluates the growth of the localities by the volume of population, through a spatial analysis, where the projections of the total population by locality are used in the period from 2010 to 2030; as well as the processes that have caused the susceptibility of the natural soil to be transformed into urban, promoting the extension of the urban spot. The results are obtained by applying the method of interpolation by isolines in the GIS (Geographic Information Systems), in which the spatial distribution of the population is established according to the typology of the localities.
\end{abstract}

Keywords: urban growth and land use. *Universidad de Ciencias y Artes de Chiapas, México. **Universidad Autónoma del Estado de Hidalgo, México. Correos electrónicos:
marcelino.garcia@unicah.mx, Ifranco@uaeh.edu.mx, joseg@uaeh.edu.mx 


\section{Introducción}

La Zona Metropolitana de Pachuca (ZMP) es una región ubicada en el estado de Hidalgo, al norte de la megalópolis de México (Valle de México). De acuerdo con SEDATU, CONAPO e INEGI (2018), la ZMP está conformada por siete municipios: Epazoyucan, Mineral del Monte, Pachuca de Soto, Mineral de la Reforma, San Agustín Tlaxiaca, Zapotlán de Juárez y Zempoala, los cuales durante los últimos veinte años han mostrado un crecimiento espacial de sus localidades y específicamente del uso del suelo para los nuevos asentamientos, cuyo resultado ha sido el proceso de transformación del espacio rural-urbano. Esto ha ocasionado una nueva reconfiguración del poblamiento y ha alterado la aptitud natural del suelo y cambio de uso ante la escasa planeación territorial regional que ha conllevado el aumento disperso y desordenado de la mancha urbana, sobre todo en los municipios centrales de la Zona Metropolitana, como Pachuca y Mineral de la Reforma.

Ante este panorama, han emergiendo de forma acelerada nuevos asentamientos urbanos, lo cual ha modificado el uso de suelo natural según la vocación física; de estos cambios dependerá la magnitud de las consecuencias del futuro por la fuerte presión dada la expansión de los servicios básicos, como la disponibilidad de agua potable por parte de la población, con lo que se debe prever la escasez de recursos que sirven de abasto para cubrir las necesidades básicas (González y Larralde, 2013).

Los cambios en la forma de apropiación del suelo a través del tiempo han tenido repercusiones en el territorio como la presión y la especulación económica inmobiliaria a través del aumento del costo del suelo urbano, cinturones de desigualdad social del costo y tenencia de la tierra (Pérez, 2018). Además, se establecen procesos sociodemográficos externos que determinan la consolidación de los espacios urbanos al interior de las manchas urbanas, como la atracción de migrantes intermunicipales e interestatales, mayor predominancia de Commuter ${ }^{1}$-siendo la conurbación el principal centro de afluencia de la $\mathrm{ZMP}-$, propensión de cinturones de desigualdad y marginación urbana -vista en la dualidad de estratificación social y morfológica dentro las urbes-, por lo que no existe ninguna metodología que determine y prevenga el crecimiento espacial de los asentamientos humanos futuros.

\footnotetext{
${ }^{1}$ El commuting se puede definir como el desplazamiento que se produce por la disociación entre lugar de trabajo y/o estudio y lugar de residencia. Es decir, el individuo cambia de lugar de residencia sin cambiar el sitio de trabajo y/o estudio y viceversa. La persona que realiza este tipo de movimiento suele denominarse commuter (García, 2010).
} 


\section{Algunos elementos para entender los cambios de uso de suelo}

La vocación o aptitud del suelo reconocida socialmente ha cambiado con el tiempo; las presiones para el cambio de uso son crecientes y en un proceso de escasa planeación cuyo propósito es mantener lo que "debe ser"; según el conocimiento tradicional, se ve afectada por diversos factores que interactúan de manera independiente, incluso, a pesar de la normatividad vigente que, por cierto, se da en un vacío institucional o, en el mejor de los casos, está incompleta, a veces contradictoria y muchas veces obsoleta o bien no es implementada porque no existen recursos para vigilar su aplicación y cuando se utiliza se hace de manera errática, dependiendo del interés coyuntural de la autoridad como principal regulador de los cambios que se realizan en el territorio (Graizbor, 2002).

Existe una fuerte presión por generar cambios y tendencias diferenciadas que afectan el uso del suelo en las grandes urbes y en los pueblos cercanos, en el medio ruralurbano y en el resto del territorio a partir del acelerado proceso de urbanización que involucra a toda la población nacional en búsqueda de mejores oportunidades y cercanía hacia los bienes y servicios; este fenómeno afecta los recursos naturales superficiales y en el subsuelo, y genera deterioro ambiental y contaminación que crece a mayor velocidad que la población en el contexto del ordenamiento territorial, por lo que es pertinente hacer una revisión de las categorías primarias y secundarias de la clasificación de los usos del suelo ahora vigente, pues habría nuevas actividades relacionadas con el manejo de los recursos y la contabilidad ambiental que los anteriores sistemas clasificatorios no contemplaban (Lezama y Dominguez, 2006).

Actualmente, prevalece una mayor presión social por controlar los espacios vacíos que con el tiempo se adoptan para otros fines y se encuentran cercanos a los centros urbanos. Las condiciones físicas del suelo se destruyen haciendo más vulnerables los cambios en la transformación del uso y aprovechamiento de los recursos (Álvarez, 2010). Estos cambios de uso de suelo con fines urbanos han incidido en la aglomeración de habitantes en territorios aislados de manera acelerada, factor de gran importancia en la configuración del poblamiento a través de localidades rurales a urbanas que agrupan mayor número de viviendas, así como la demanda por ampliar la infraestructura física e hidráulica que requiere la sociedad para satisfacer sus necesidades de bienestar (Cifuentes y Londoño, 2010). Finalmente, los cambios generados por la población urbana permitirán mantener un control sobre la dinámica en el uso de suelo y el ordenamiento de la ciudad, así como plantear situaciones probables para mitigar y adaptar en políticas públicas que favorezcan el uso y la transformación del suelo. 


\section{Crecimiento de las localidades en la ZMP}

El proceso de formación de la Zona Metropolitana se ha basado en una organización espacio-temporal, la cual, al estar expuesta por la presión sociodemográfica en la región central del país, es decir, su cercanía con la Ciudad de México, generó el establecimiento de una reorganización geográfica del municipio de Pachuca con los municipios colindantes. De acuerdo con el grupo interinstitucional SEDATU, CONAPO, INEGI (2018), los criterios para establecer el proceso metropolitano se definen de la siguiente forma:

- Municipios centrales y conurbación física: Pachuca y Mineral de la Reforma.

- Distancia e integración funcional de carácter urbano: Mineral del Monte.

- Planeación y Política-Urbana: Epazoyucan, San Agustín Tlaxiaca, Zapotlán de Juárez, Zempoala.

Según la clasificación de INEGI (Villalvazo et al., 2002), el estado de Hidalgo aún mantiene una población mayormente rural (agrupada en localidades menores a 2,500 habitantes) al norte y a menos de $100 \mathrm{~km}$ de la Ciudad de México; es una región de mayor crecimiento urbano que durante las últimas dos décadas ha definido el ritmo y la orientación del proceso de urbanización social en México (Granados, 2010).

Los municipios con mayor impacto son aquellos donde existe una alta concentración de localidades, tal es el caso de la conurbación de Pachuca y Mineral de la Reforma; respecto al crecimiento de las localidades, en 1990, las rurales representaban el $96 \%$, y en los siguientes 20 años disminuyeron a 93\%, mientras que las urbanas presentaron un aumento del $3 \%$ para ese mismo periodo, resultado del proceso de urbanización, el cual se ha dado en ciertas zonas y no ha sido homogéneo (ver figura 1).

Los municipios centrales han concentrado la población urbana. Pachuca de Soto, al no contar con mayor espacio para urbanizar, ha desbordado su crecimiento hacia otros espacios contiguos, como el municipio de Mineral de la Reforma, impactando en las localidades rurales cercanas e incrementando las urbanas para usar de zona habitación los nuevos asentamientos sobre la frontera municipal. Por otro lado, mientras algunas localidades rurales desaparecieron en 2010, otras emergieron duplicándose en tan sólo 20 años. Por su parte, en Mineral del Monte y San Agustín Tlaxiaca, su número de localidades rurales sigue siendo mayor con respecto a las urbanas. Finalmente, en Zapotlán de Juárez y Zempoala, la distancia geográfica con el centro de la Zona Metropolitana ha generado un aumento de localidades urbanas y mayor desarrollo de infraestructura de conectividad intermunicipal dentro de la ZMP. 
Figura 1. Tipología de las localidades en la ZMP de 1990 a 2010

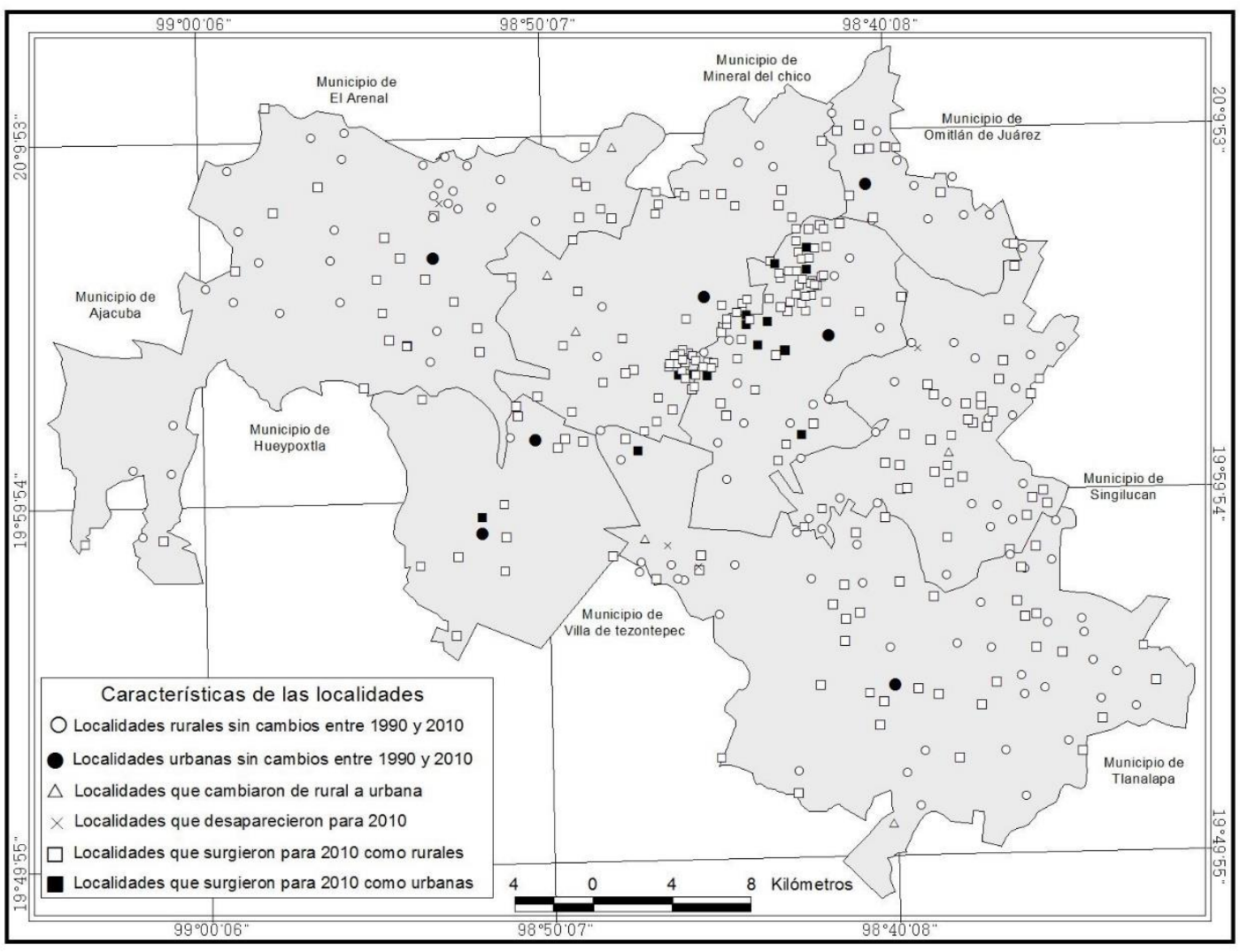

Fuente: elaboración propia con base en INEGI (1990, 2000 y 2010a).

Los municipios de Epazoyucan y Zempoala presentan un aumento en las localidades del 50\% del total con respecto a las que tenía en 1990; en el caso del San Agustín Tlaxiaca, este incremento fue del 35\% en la cantidad de localidades, por lo que en general existe una evolución en la construcción espacial de éstas dentro de la Zona Metropolitana de Pachuca (ver cuadro 1).

El crecimiento de las localidades rurales y urbanas en el periodo 1990-2010 ha sido una constante en el proceso de metropolización de la ciudad de Pachuca de Soto, debido a que presenta cambios en la dinámica y en la estructura de la población de las localidades (Tovar, 2011). Algunos municipios han incrementado hasta un $84 \%$ la cantidad de localidades, como Mineral de la Reforma, pues, al no existir más espacio físico urbanizable en Pachuca, ha conllevado un aumento muy sustancial en el municipio contiguo para el año 2010. Por su parte, Zapotlán de Juárez también ha implicado un cambio importante en sus localidades en 1990, pues sólo tenía dos localidades y para 2010 se incrementó notablemente hasta llegar a tener seis veces más. 
Cuadro 1. Clasificación por tipos de localidad en la ZMP

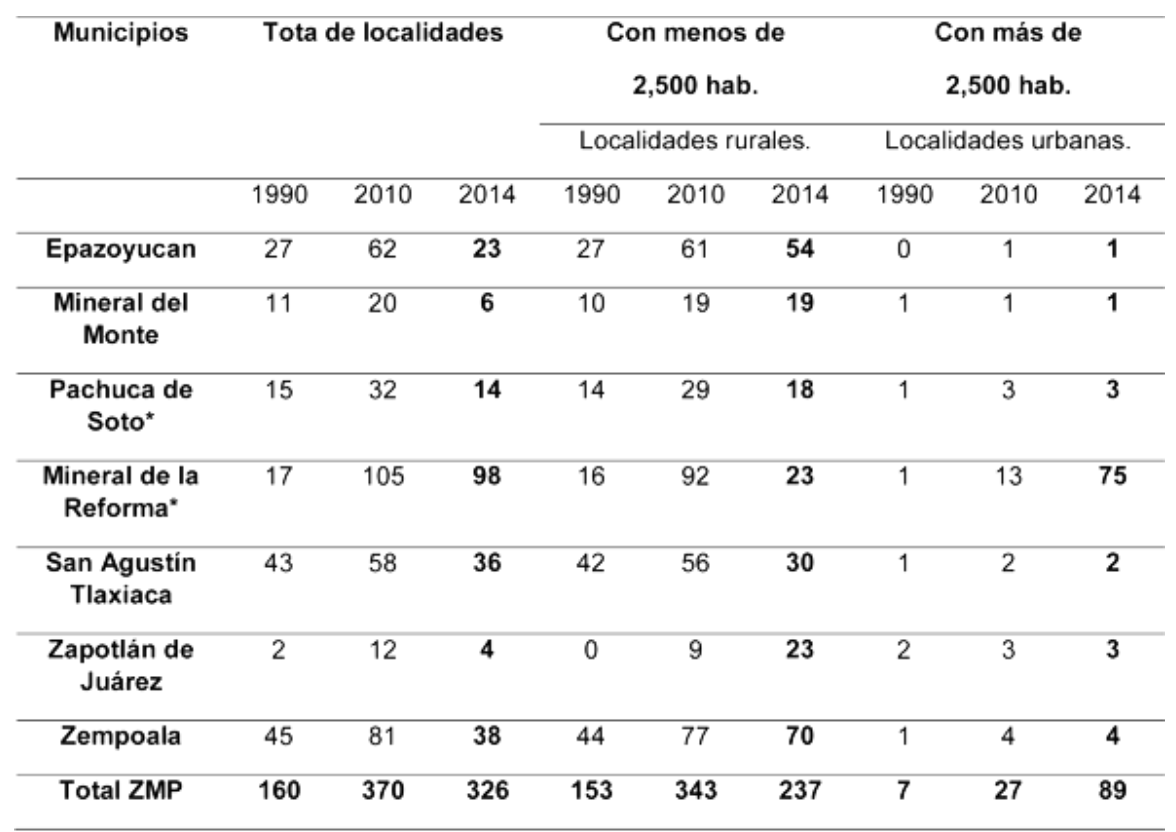

Fuente. elaborado a partir de los datos de INEGI, 1990, 2005a, 2014. *Municipios centrales.

\section{Uso de suelo 1997-2016}

La aptitud del suelo es su capacidad natural modificada para soportar un uso definido, y depende principalmente de las características biofísicas y ambientales de cada ecosistema terrestre; por tanto, esta aptitud puede ser modificada por acciones humanas a través del cambio de uso de suelo, el cual ha sido el principal elemento ambiental que sufre alteraciones en el territorio (Balbo, et. al 2003; López et al., 2015).

Alrededor del 54\% de la población mundial actual reside en áreas urbanas y se prevé que para 2050 llegará al 66\%, según la ONU (Naciones Unidas, 2014). Los cambios de uso de suelo durante el siglo XX han surgido como un proceso de diferentes escalas espaciales con consecuencias en el territorio; una de las principales causas de estos cambios de uso, a niveles local, regional y global, sin duda, es la presión directa e indirecta que ejerce el hombre sobre los recursos naturales de manera local, cuyos resultados son: pérdida y degradación de los suelos, cambios en el microclima y pérdida de la biodiversidad de especies a nivel regional, los cuales afectan el funcionamiento de cuencas hidrográficas y de asentamientos humanos; y a nivel global coadyuvan a las emisiones de gases efecto invernadero (Delgado et al., 2017).

El suelo es un bien heterogéneo finito e inamovible aunque sustituible a otros factores socioeconómicos (trabajo o capital); bajo ciertas condiciones, ciertos terrenos o lugares, son más deseables que otros por razones geográficas y por su composición físicobiológica, es decir, depende de las actividades que allí se realizan o se localizan, y el precio 
del suelo es modificable según las necesidades o intereses (Martínez, 2006). En la ZMP se presentan diez tipos de usos de suelos predominantes de acuerdo con la carta de uso de suelo y vegetación generada por INEGI (1997). Sin embargo, los tipos de cultivo específico que se aplican en cada unidad agrícola al interior de los municipios pueden variar por las condiciones propias de los ejidatarios o grupo de campesinos encargados de realizar las tareas de esta actividad (ver cuadro 2).

Cuadro 2. Tipos de usos de suelo predominantes en la ZMP

\begin{tabular}{|c|c|}
\hline TIPOS DE USO DE SUELO & CARACTERISTICAS \\
\hline Agricultura de Riego anual & $\begin{array}{l}\text { Áreas de cultivo que presentan ciclos de siembra continuos } \\
\text { a lo largo del año; demanda un sistema de humedad de tipo } \\
\text { inducido de canales de agua rodada que es acumulada en } \\
\text { presas hidrológicas y en ocasiones por la extracción de agua } \\
\text { subterránea mediante pozos profundos. }\end{array}$ \\
\hline Agricultura de Temporal & $\begin{array}{l}\text { Áreas de cultivo que dependen de las condiciones de } \\
\text { humedad meteorológica para su siembra a lo largo del año. }\end{array}$ \\
\hline $\begin{array}{l}\text { Agricultura de Temporal y Pastizal } \\
\text { inducido }\end{array}$ & $\begin{array}{l}\text { Áreas de cultivo que son utilizadas para la siembra de pasto } \\
\text { utilizado para dar sustento a la cría de ganado. }\end{array}$ \\
\hline Bosque & $\begin{array}{l}\text { Áreas de distintas variedades de arbustos, ubicadas a más } \\
\text { de } 2500 \mathrm{msnm} \text {, localizadas en la parte montañosa que } \\
\text { circunda la zona norte del área metropolitana. }\end{array}$ \\
\hline Cuerpo de Agua Perenne interior & $\begin{array}{l}\text { Áreas lagunares o encharcamientos naturales, que se } \\
\text { presentan al interior de la zona metropolitana. }\end{array}$ \\
\hline Chaparral & $\begin{array}{l}\text { Árboles bajos, espinosos, que se presentan en áreas } \\
\text { semiáridas, carentes de vegetación secundaria y se asocia } \\
\text { a suelos arenosos o con carencia de materia orgánica. }\end{array}$ \\
\hline Matorral & $\begin{array}{l}\text { Áreas con árboles de tamaño mediano, con vegetación } \\
\text { secundaria circundante, con suelos ricos en materia } \\
\text { orgánica y pueden incorporarse a las zonas de agricultura o } \\
\text { ganadería extensiva. }\end{array}$ \\
\hline Pastizal Inducido & $\begin{array}{l}\text { Areas de cultivo especifico de siembra de pasto utilizada } \\
\text { para las distintas especies de cria de ganado a escala } \\
\text { familiar. }\end{array}$ \\
\hline Áreas sin vegetación aparente & $\begin{array}{l}\text { Áreas que no presentan un tipo de vegetación aparente o en } \\
\text { su caso carecen de plantas. }\end{array}$ \\
\hline Uso Urbano & $\begin{array}{l}\text { Suelo transformado para fines de construcción de viviendas } \\
\text { o actividades económicas. }\end{array}$ \\
\hline
\end{tabular}

Fuente: INEGI (2001 y 2014a).

\section{Proyecciones del crecimiento de la población urbana}

El comportamiento de la población total por localidad en la ZMP se ha mantenido estable en los municipios que presentan predominio de población rural, como Mineral del Monte y Epazoyucan, los cuales en 2005 alcanzaron un máximo de crecimiento poblacional; sin embargo, las proyecciones de población realizadas por CONAPO para el periodo 20052030 señalan una tendencia a disminuir. 
Por otro lado, las localidades de Zapotlán de Juárez, Zempoala, San Agustín Tlaxiaca muestran un crecimiento poblacional de 5 a $10 \%$ por quinquenio pasando de rurales a urbanas. Pachuca de Soto presenta un ritmo menor que Mineral de la Reforma, que muestra una tendencia más elevada del $10 \%$ en 20 años. En conjunto, mantienen un acelerado proceso de urbanización y agrupan mayor volumen de población hacia 2030 (ver cuadro 3).

Cuadro 3. Proyecciones de población total en las localidades de la ZMP de $\mathbf{2 0 1 0}$ a $\mathbf{2 0 3 0}$

\begin{tabular}{|c|c|c|c|c|c|c|c|}
\hline $\begin{array}{c}\text { Nombre de la } \\
\text { localidad }\end{array}$ & Municipio & 2005 & 2010 & 2015 & 2020 & 2025 & 2030 \\
\hline Epazoyucan & Epazoyucan & 2,601 & 2,464 & 2,282 & 2,107 & 1,934 & 1,774 \\
\hline Mineral del Monte & $\begin{array}{l}\text { Mineral del } \\
\text { Monte }\end{array}$ & 9,832 & 8,833 & 7,847 & 6,932 & 6,093 & 5,325 \\
\hline Pachuquilla & $\begin{array}{l}\text { Mineral de la } \\
\text { Reforma }\end{array}$ & 4,664 & 6,581 & 8,505 & 10,364 & 12,127 & 13,751 \\
\hline $\begin{array}{l}\text { Azoyatla de Ocampo } \\
\text { (Azoyatla) }\end{array}$ & $\begin{array}{l}\text { Mineral de la } \\
\text { Reforma }\end{array}$ & 1,313 & 1,853 & 2,394 & 2,917 & 3,414 & 3,871 \\
\hline Santa Maria la Calera & $\begin{array}{l}\text { Mineral de la } \\
\text { Reforma }\end{array}$ & 1,245 & 1,757 & 2,271 & 2,767 & 3,238 & 3,671 \\
\hline El Venado & $\begin{array}{l}\text { Mineral de la } \\
\text { Reforma }\end{array}$ & 975 & 1,376 & 1,778 & 2,166 & 2,535 & 2,874 \\
\hline La Providencia & $\begin{array}{l}\text { Mineral de la } \\
\text { Reforma }\end{array}$ & 12,482 & 17,612 & 22,761 & 27,735 & 32,455 & 36,800 \\
\hline El Chacón & $\begin{array}{l}\text { Mineral de la } \\
\text { Reforma }\end{array}$ & 1,312 & 1,851 & 2,392 & 2,915 & 3,411 & 3,868 \\
\hline $\begin{array}{l}\text { Fraccionamiento la } \\
\text { Reforma }\end{array}$ & $\begin{array}{l}\text { Mineral de la } \\
\text { Reforma }\end{array}$ & 1,295 & 1,828 & 2,362 & 2,878 & 3,368 & 3,819 \\
\hline Pachuca & $\begin{array}{l}\text { Mineral de la } \\
\text { Reforma }\end{array}$ & 36,709 & 51,794 & 66,941 & 81,569 & 95,449 & 108,228 \\
\hline $\begin{array}{l}\text { Fraccionamientos del } \\
\text { Sur }\end{array}$ & $\begin{array}{l}\text { Mineral de la } \\
\text { Reforma }\end{array}$ & 4,375 & 6,173 & 7,978 & 9,722 & 11,376 & 12,899 \\
\hline Pachuca de Soto & $\begin{array}{l}\text { Pachuca de } \\
\text { Soto }\end{array}$ & 268,945 & 295,338 & 317,801 & 335,619 & 348,877 & 357,275 \\
\hline $\begin{array}{l}\text { San Miguel Cerezo (El } \\
\text { Cerezo) }\end{array}$ & $\begin{array}{l}\text { Pachuca de } \\
\text { Soto }\end{array}$ & 1,964 & 2,157 & 2,321 & 2,451 & 2,548 & 2,609 \\
\hline Santiago Tlapacoya & $\begin{array}{l}\text { Pachuca de } \\
\text { Soto }\end{array}$ & 2,586 & 2,840 & 3,056 & 3,227 & 3,355 & 3,436 \\
\hline San Agustin Tlaxiaca & $\begin{array}{l}\text { San Agustin } \\
\text { Tlaxiaca }\end{array}$ & 9,508 & 10,473 & 11,387 & 12,211 & 12,918 & 13,498 \\
\hline Ixcuinquitlapilco & $\begin{array}{l}\text { San Agustin } \\
\text { Tlaxiaca }\end{array}$ & 2,023 & 2,228 & 2,423 & 2,597 & 2,748 & 2,872 \\
\hline San Juan Tilcuautla & $\begin{array}{l}\text { San Agustín } \\
\text { Tlaxiaca }\end{array}$ & 2,199 & 2,423 & 2,634 & 2,823 & 2,988 & 3,122 \\
\hline Zapotlán de Juárez & $\begin{array}{l}\text { Zapotlán de } \\
\text { Juárez }\end{array}$ & 8,704 & 9,384 & 9,979 & 10,477 & 10,878 & 11,164 \\
\hline Acayuca & $\begin{array}{l}\text { Zapotlán de } \\
\text { Juárez }\end{array}$ & 7,840 & 8,453 & 8,988 & 9,437 & 9,798 & 10,056 \\
\hline Zempoala & Zempoala & 5,701 & 6,254 & 6,738 & 7,138 & 7,457 & 7,680 \\
\hline Santo Tomás & Zempoala & 1,957 & 2,148 & 2,314 & 2,450 & 2,559 & 2,637 \\
\hline $\begin{array}{l}\text { Jagüey de Téllez } \\
\text { (Estación Téllez) }\end{array}$ & Zempoala & 3,013 & 3,306 & 3,561 & 3,772 & 3,940 & 4,059 \\
\hline $\begin{array}{l}\text { Santiago } \\
\text { Tepeyahualco }\end{array}$ & Zempoala & 2,337 & 2,564 & 2,762 & 2,926 & 3,056 & 3,149 \\
\hline \multicolumn{2}{|l|}{ Total de población ZMP } & 393,580 & 449,690 & 501,475 & 547,200 & 586,522 & 618,437 \\
\hline
\end{tabular}

Fuente: elaboración propia con base en CONAPO (2005). 
La información publicada por CONAPO en las proyecciones de poblaciones para los municipios del país en los próximos 20 años refieren que en la ZMP la tendencia de población urbana aumentará con respecto a la población rural, además de que esta última migrará hacia zonas urbanas caracterizadas por una concentración de las actividades socioeconómicas principales. Otro problema es el aumento en la demanda de servicios públicos básicos, como la disponibilidad de agua potable para cubrir las necesidades de la población emigrante que llegue a residir en esta región del país.

Los cambios poblacionales (incremento o decremento de la población por municipio) en porcentaje referido a partir de los datos de proyecciones municipales 20052030 realizados por CONAPO, a manera de conocer la dinámica poblacional en los siguientes quinquenios, se calculó de la siguiente manera:

\section{PorVar $=$ PobProy - PobBase $* 100$}

Donde:

PorVar $=$ Porcentaje de Varación

PobProy $=$ Población Proyectada

PobBase $=$ Población Base

Dado el resultado de la población municipal proyectada en la ZMP, se estima que, para el periodo de 20 años, la población de Epazoyucan estará reducida en promedio en un $7.9 \%$, mientras que para el municipio de Mineral del Monte será del 12\%, por lo que estos municipios seguirán presentando procesos migratorios, principalmente hacia los centrales de la ZMP (ver cuadro 4).

Cuadro 4. Variación de la población total proyectada por municipio para el periodo 2010-2030
\begin{tabular}{lcccccc}
\hline Municipios & $\mathbf{2 0 1 0 ^ { \star }}$ & $\mathbf{2 0 1 5}$ & $\mathbf{2 0 2 0}$ & $\mathbf{2 0 2 5}$ & $\mathbf{2 0 3 0}$ & Promedio \\
\hline Epazoyucan & 100 & -7.4 & -7.7 & -8.0 & -8.3 & $\mathbf{- 7 . 9}$ \\
\hline Mineral del Monte & 100 & -11.2 & -11.7 & -12.1 & -12.6 & $\mathbf{- 1 1 . 9}$ \\
\hline Pachuca de Soto & 100 & 7.6 & 5.6 & 4.0 & 2.4 & $\mathbf{4 . 9}$ \\
\hline Mineral de la Reforma & 100 & 29.2 & 21.9 & 17.0 & 13.4 & $\mathbf{2 0 . 4}$ \\
\hline San Agustín Tlaxiaca & 100 & 8.7 & 7.2 & 5.8 & 4.5 & $\mathbf{6 . 6}$ \\
\hline Zapotlán de Juárez & 100 & 6.3 & 5.0 & 3.8 & 2.6 & 4.4 \\
\hline Zempoala & 100 & 7.7 & 5.9 & 4.4 & 3.0 & $\mathbf{5 . 3}$ \\
\hline Promedio en la ZMP \% & $\mathbf{5 . 9}$ & $\mathbf{3 . 7}$ & $\mathbf{2 . 1}$ & $\mathbf{0 . 7}$ & $\mathbf{3 . 1}$ \\
\hline
\end{tabular}

Fuente: elaboración propia con base en CONAPO (2005). *Se considera la población total como base para definir el porcentaje de variación posterior para los siguientes quinquenios. 
El municipio de Pachuca presenta una disminución quinquenal, en promedio de 5\%; por consiguiente, en 2015 alcanzaron su máximo crecimiento poblacional; en los posteriores quinquenios, el crecimiento disminuirá paulatinamente hasta quedar en $2.5 \%$ para 2030. El caso más importante de crecimiento es el municipio de Mineral de la Reforma, siendo el más alto en la Zona Metropolitana con cerca de 30\% en 2015, pero también es el de mayor margen con 17\%; la disminución más baja será en el quinquenio de 2030 con tan sólo el 13\% del crecimiento. En casos similares se ubican los municipios de San Agustín Tlaxiaca, Zapotlán de Juárez y Zempoala donde los datos muestran que su crecimiento máximo en 2015 fue entre el 6 y el 9\%, mientras que con el paso de los años disminuirá y en el año 2030 establecerá un crecimiento del 4 al 6.5\%.

Existen localidades urbanas que son cabeceras municipales, como Epazoyucan y Mineral del Monte, y que a partir de 2015 presentan una disminución en su población total, lo cual puede justificar el incremento de las localidades rurales y urbanas en Mineral de la Reforma, ubicadas de manera contigua a la mancha urbana de Pachuca y que para el año 2010 han visto un crecimiento desmedido.

\section{Materiales y métodos}

\section{Datos estadísticos}

Para la identificación de las localidades existentes en los municipios de la ZMP, se utilizó el Catálogo de localidades del Censo de Población y Vivienda de 1990 y 2010; posteriormente, se empleó el documento Características de las localidades (INEGI, 2014b), con el fin de actualizar y analizar los tipos de localidad en la Zona Metropolitana, lo cual generó la base empírica de la evolución de las localidades en la metrópoli; algunos casos, por cuestiones de la dinámica de la población desaparecieron, pero se crearán nuevos en uno o más municipios.

Asimismo, se utilizaron las proyecciones de población generadas por el Consejo Nacional de Población en 2005 para 2010-2030 a escala de localidad. En este documento se realizó una estimación del crecimiento natural que presenta la dinámica demográfica en periodos quinquenales. Para el cálculo de estas estimaciones, se utilizaron variables, como: población total, natalidad, mortalidad, emigración y migración para un tiempo específico, las cuales se pueden describir en la siguiente ecuación: 


$$
P P=P i+(N a c-D e f)+(I n m-E m i)
$$

Donde:

PP = Población Proyectada

$\mathrm{Pi}=$ Población inicial

Nac $=$ Nacimiento

Def $=$ Defunciones

Inm $=$ Inmigraciones

Emi $=$ Emigraciones

Cartas de uso de suelo y vegetación

El trabajo identifica y evalúa la susceptibilidad de los tipos de suelo a sufrir una transformación con fines distintos al de su aptitud física y química mediante el cálculo del tamaño de áreas geoespaciales; para ello, se han utilizado fotografías áreas e imágenes de satélite en distintas temporalidades, con las cuales se han elaborado las "cartas de uso de suelo y vegetación" (Cano et al., 2016). Con este proceso y enfoque general, se elaboró la primera carta de uso de suelo a finales de los años ochenta y a principios de los noventa denominada Serie I (INE e INEGI, 1997).

Con el apoyo de los Sistemas de Información Geográfica (SIG), se han identificado las áreas de suelo que han sido transformadas progresivamente en el tiempo para fines urbanos en los asentamientos humanos consolidados. Sin embargo, para el 1998 se hizo una actualización de la carta de uso de suelo y vegetación. En ésta aparecen las manchas urbanas de la Zona Metropolitana; la información ha servido de referencia para elaborar la "Carta de uso actual del suelo y vegetación", denominada Serie II, que contiene las manchas urbanas de 1990 a 1998 (INEGI, 2001) (ver figura 2).

Posteriormente, INEGI (2005b) actualizó la carta de uso de suelo y vegetación en la Serie III, pero para fines de esta investigación no se observaron cambios significativos; el tamaño de las manchas urbanas se actualizó en el estudio "Delimitación de las Zonas Metropolitanas de México". Para 2010, se realizó otra actualización denominada Serie IV, basada en el XIII Censo de Población y Vivienda, en donde se presentan las manchas urbanas por localidad en los municipios que integran la ZMP. 
Figura 2. Uso del suelo y vegetación en la ZMP en 1990

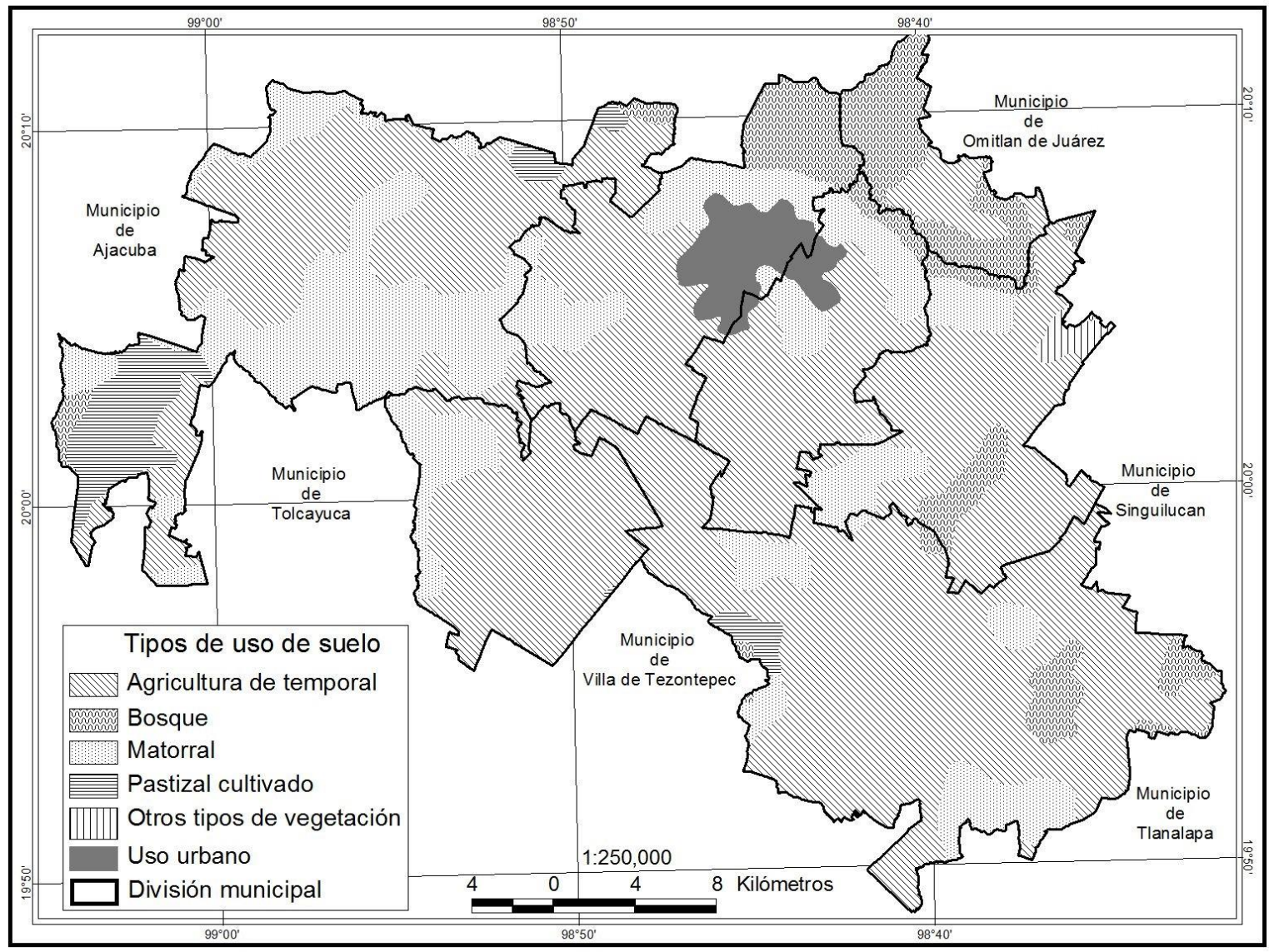

Fuente: elaboración propia con datos de INE (1997).

En los siguientes años se elaboraron dos cartas más, la Serie V en INEGI (2013) y la Serie VI con un conjunto de datos vectoriales sobre uso de suelo y vegetación; se actualizó en 2016. Esta información se obtuvo a partir de la aplicación de técnicas de fotointerpretación con imágenes de satélite Landsat TM8 seleccionadas en 2014 y ha servido de referencia en la investigación como última fuente de consulta sobre el incremento de las manchas urbanas de las localidades que integran la ZMP (Cano et al., 2016; INEGI, 2016) (ver figura 3). 
Figura 3. Uso del suelo y vegetación en la ZMP en 2016

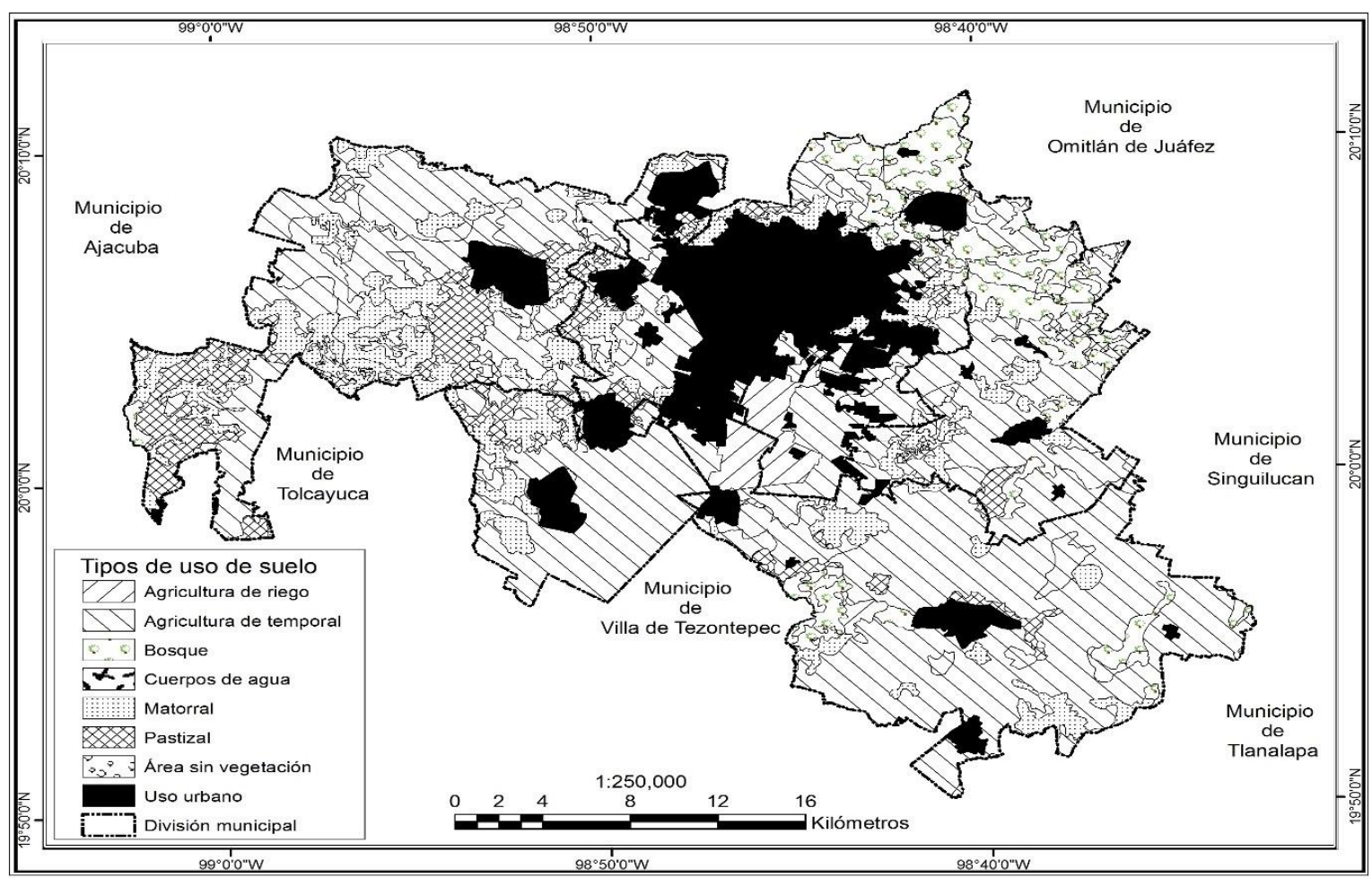

Fuente: elaboración propia con datos de INEGI (2010b, 2016).

\section{Aplicación SIG}

La distribución del uso de suelo se puede calcular mediante cualquier sistema de información geográfica (SIG); como primer paso, se debe realizar una referencia geográfica de la capa para obtener la cantidad de $\mathrm{km}^{2}$ que ocupa cada polígono de cada uso en cada municipio, es decir, las dimensiones de las manchas urbanas de 1998 hasta 2016; este dato es de gran importancia, ya que puede evaluar el incremento en el uso de suelo urbano para otros distintos usos, como agricultura de riego anual, agricultura de temporal, agricultura de temporal y pastizal inducido, áreas sin vegetación aparente, bosque, chaparral, cuerpo de agua perenne interior, matorral, pastizal inducido y suelo urbano (ver cuadro 5).

La importancia de incluir este tipo de metodología (los SIG) en estudios demográficos y urbanos permite la representación cartográfica a través de isolíneas, tomando como referencia los datos de población total a escala de localidades representadas en puntos, las cuales, al representarse en el espacio geográfico, generá isolíneas con valores similares (rango similar las junta y rangos de mayor valor a la media los aleja) como una forma de explicar el comportamiento de la población de un territorio (Pascual, et. al 2010). 
El método de interpolación split es una aplicación que está incorporada en cualquier sistema de información y permite calcular la distribución espacial de la población total mediante un punto que representan las localidades de un territorio, como ocurre a nivel de Zona Metropolitana. La ventaja de utilizar este proceso técnico con datos demográficos es la identificación con isolíneas de las áreas de concentración de población, las cuales se traducen en zonas de probable incremento en los asentamientos poblacionales; sin embargo, si se le asocian puntos de representación con datos de estimaciones de población futura, el resultado permite corroborar que la tendencia de crecimiento se establecerá en esos puntos de referencia, dibujados en mapas de isolineas (ver figura 4).

Figura 4. Distribución de la población en 2010 y proyección de la población por localidades urbanas en la ZMP de $\mathbf{2 0 1 0}$ a 2030

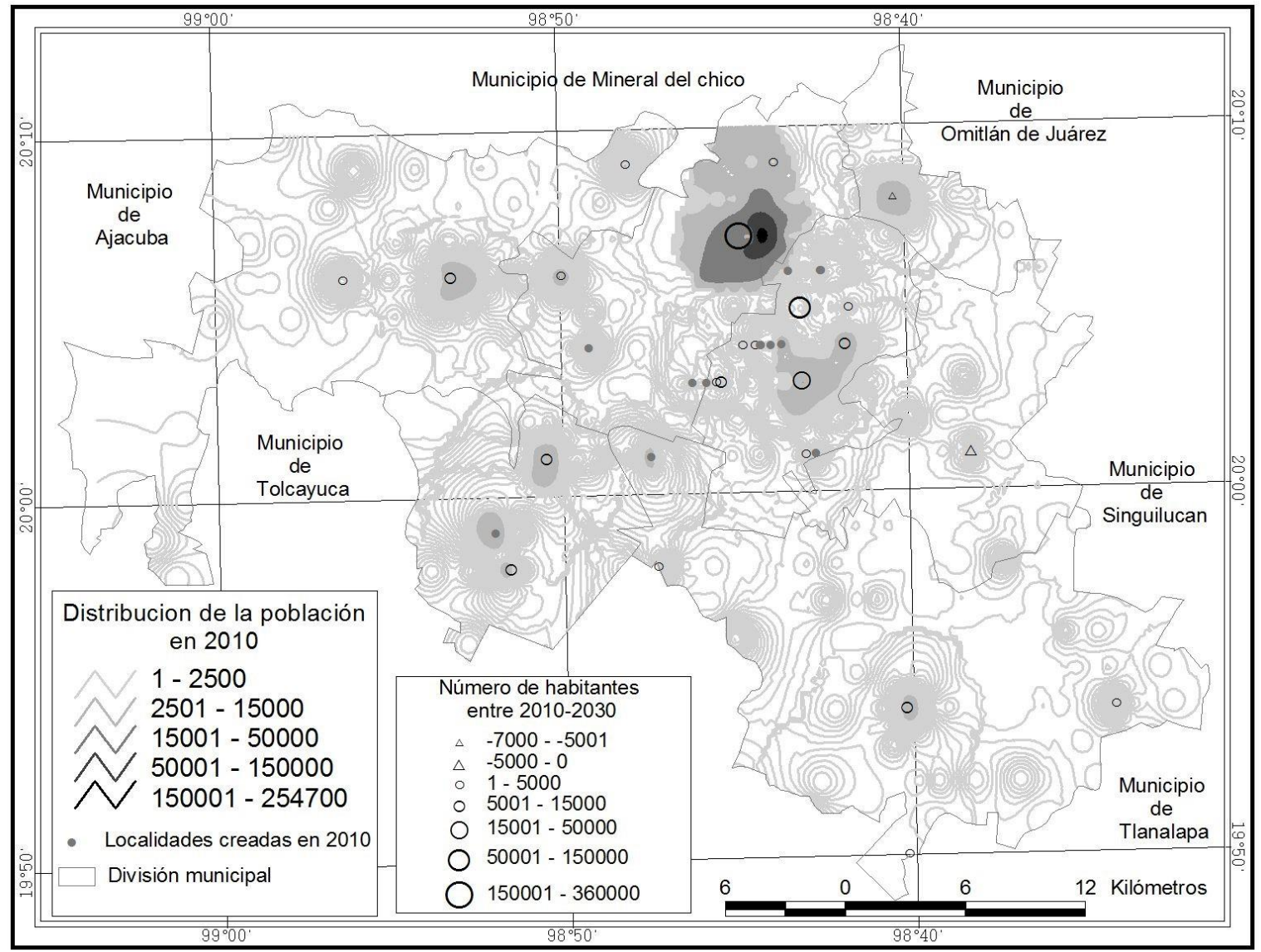

Fuente: elaboración propia con datos de CONAPO (2005) e INEGI (2010). 


\section{Resultados}

El municipio de Epazoyucan cuenta con una superficie total de 139.79 km²; el uso de suelo predominante es apropiado para la agricultura de temporal con el $70 \%$ de la superficie total de 1997 a 2016. Aunque el área urbana creció sólo un 2.16 km², representa el 5\% del territorio municipal en el mismo periodo. Mineral del Monte tiene una superficie de 53.05 $\mathrm{km}^{2}$, de los cuales, 73.5\% ha mantenido una vocación natural de distintos tipos de bosque, por lo que su área urbana equivale al 9.2\% de su superficie total (ver cuadro 5).

Para el municipio de Mineral de la Reforma, la superficie total fue de $113.19 \mathrm{~km}^{2}$; en 1997; cabe señalar que el uso predominante está destinado para agricultura de temporal en un $85 \%$ de la superficie; en 2005 disminuyó un $11 \%$ y en 2016 fue de 38\%. La disminución territorial de este tipo de uso de suelo se ha convertido en áreas de urbanización que han crecido de manera intensiva en dos décadas: 0.2\% en 1997 y en 2016 alcanzó el 33 \% de la superficie municipal. En Pachuca la superficie total corresponde a 152.96 km² y presentó un tipo de uso de suelo con vocación de agricultura de temporal y pastizal inducido con el 64\% de su superficie en 1997; para los siguientes años, disminuyó al $60 \%$ de la superficie, la cual fue transformándose en uso de suelo urbano y el crecimiento de la mancha urbana alcanzó el 47\% de la superficie total municipal en 2016 (ver cuadro 5).

En el municipio de San Agustín Tlaxiaca, la superficie total es de 295.19 km²; el uso de suelo predominante para agricultura de temporal en 1997 representó el 68\%; aunque en 2016 esta superficie disminuyó un 20\%, ha sido transformada en uso de suelo urbanizado; por lo que en 2016 representó el 6.2\% de la superficie municipal total. En el municipio de Zapotlán de Juárez, la superficie total fue de 128.09 km²; en 1997, la superficie destinada a la agricultura de temporal representó el 90\%, y para 2016 ésta disminuyó un 23\%, y se urbanizó sólo el 11\% y el resto se utilizó para cambiar su vocación natural a pastizal inducido.

Por último, en Zempoala, la superficie total es de $317.64 \mathrm{~km}^{2}$; el $81.5 \%$ se destinó a la agricultura de temporal y, en 2016, sólo presentaba un 7.2\% menos, de los cuales la superficie con uso de suelo urbano es cerca del $4.5 \%$ del total de la superficie municipal (ver cuadro 5). 
Cuadro 5. Cuantificación de los tipos de uso de suelo en la ZMP 1997-2016

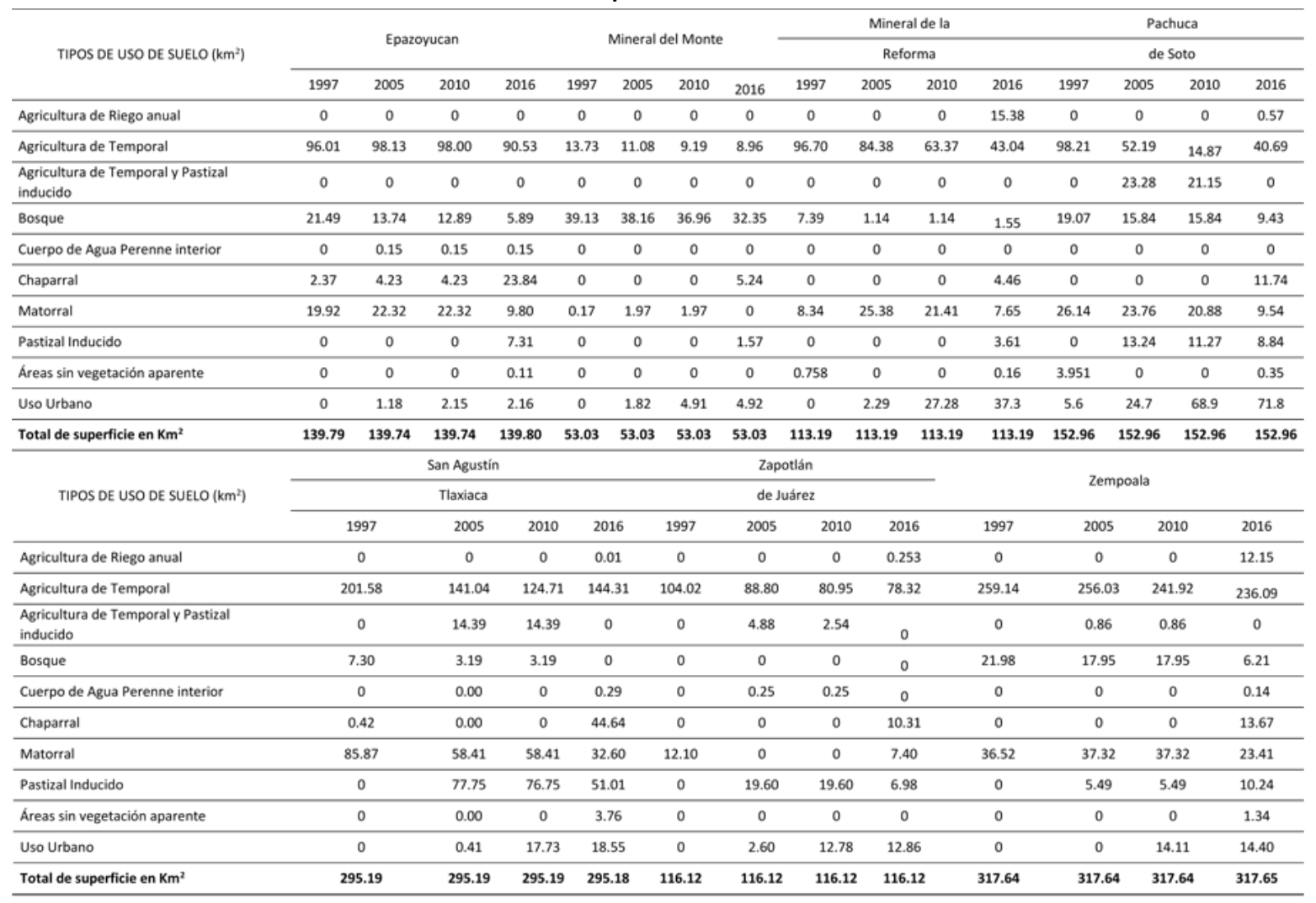

Fuente: elaboración propia a partir de los datos calculados mediante SIG de la carta de uso actual del suelo y vegetación Series I, III, IV y VI (INEGI).

El uso de suelo destinado para agricultura de temporal con pastizales inducidos se encuentra en la localidad de Acayucan en el municipio de Zapotlán de Juárez, el cual podrá expandirse hacia el norte por la cercana frontera territorial que muestra con el municipio de Pachuca, al ser una zona con características físicas aptas para los asentamientos urbanos; la mancha urbana se puede acrecentar con el tiempo, lo cual conllevaría a perder terrenos que, con la falta de una producción mínima, no soportaría la presión sociodemográfica que influirá en la construcción de nuevas viviendas y modificaría el uso de suelo en esa región de la Zona Metropolitana.

El uso de suelo de bosque define una tendencia de modificación en los municipios de Epazoyucan y Mineral del Monte de acuerdo con la cantidad población rural que habita en esta región, debido a que presenta una dinámica demográfica migratoria hacia zonas más urbanas; los datos estadísticos refieren un ritmo de crecimiento poblacional estable en estos municipios. Lo anterior permite deducir que el manejo ambiental en este uso de suelo será de mínimo impacto. Aunque presentan una disminución en la presión por el uso de suelo urbano, mediante programas de conservación se pueden rescatar estas zonas y así aprovechar los recursos de una manera más sustentable. 
Se prevé que el crecimiento de la población se mantendrá estable en las localidades ubicadas en la parte sur del municipio de Pachuca en dirección hacia el de Mineral de la Reforma y hacia el sur colindando con la ZMVM, manifestándose hacia esta parte el crecimiento urbano en las siguientes décadas. El uso más afectado por el crecimiento urbano hacia la parte sur-sureste y suroeste es el de agricultura de temporal, aunque también se ven afectadas algunas otras áreas donde se produce pastizales inducidos para actividades pecuarias; sin embargo, en la parte norte de la mancha urbana se expande el uso de suelo de matorral, el cual se ve afectado por este proceso de expansión.

Otro tipo de uso de suelo que presenta una mayor presión de modificación hacia el futuro es el pastizal inducido en la parte sur del municipio de San Agustín Tlaxiaca y al oeste de Pachuca, donde, con el pasar de los años, la mancha urbana se podría expandir de manera muy lenta pero constante ocupando una superficie menor a la mostrada en 2016.

Aunque existen localidades que presentan mayor crecimiento poblacional para el año 2010, no se encuentran reflejadas en el cálculo de proyección de la población realizado por CONAPO para el periodo 2005-2030, pues se muestra que no todas las localidades urbanas están proyectadas hacia esta temporalidad; la aplicación del Censo de Población y Vivienda en 2010 realizado por INEGI refiere que existen localidades rurales en 2005 que se han modificado para convertirse en población urbana de acuerdo con el criterio definido anteriormente y se puede observar la distribución de la población por localidad urbana, donde se describen las zonas de crecimiento de población para el periodo 20102030 a través de puntos que muestran el rango de población que alcanzarán en los próximos veinte años.

\section{Conclusiones}

Los municipios centrales de la ZMP se encuentran en un proceso dinámico en cuanto al crecimiento poblacional; sin embargo, no ha sido un factor que dé estabilidad a la estructura poblacional formada a través de los años recientes, manifestada como una zona atractiva para la población que reside en el centro del país, pues la población urbana ha migrado hacia esta región. Esto repercute en mejorar las condiciones de vida actuales y en incentivar la realización de una planeación urbana con una visión hacia los posibles escenarios que garanticen estabilidad social, económica y ambiental a fin de impulsar a la región como un centro de desarrollo integral en una economía dinámica que beneficie a todos los sectores involucrados.

Con relación a los datos estadísticos de los censos de población, se han elaborado proyecciones de población de las localidades entre 2010 y 2030, aunque en 2018 se ha ampliado hasta 2050. Esta información permite establecer el probable crecimiento y la presión demográfica que presentará un área geográfica en un tiempo y sirve para tomar decisiones en los diferentes sectores sociales y administrativos de los distintos niveles de 
gobierno y en aquellas áreas susceptibles a sufrir transformaciones de ampliación como infraestructura o de servicios. Este trabajo concluye que la población urbana se encuentra mayormente consolidada a través de las localidades, sin embargo, debe propiciar redes de desarrollo hacia las zonas periféricas de manera ordenada y con inversión a largo plazo; estos cambios se pueden prever con estudios que permitan construir una mayor visión que identifique las factibilidades del desarrollo metropolitano.

El municipio de Pachuca alcanzará su máximo de crecimiento urbano y poblacional no más allá del año 2015, lo que aumentará el crecimiento de la mancha urbana hacia los municipios del sur de la ZMP; con el paso de los años se han establecido centros de poblamiento a través de localidades que demandan servicios públicos, lo cual hace que se tenga una mayor presión por el manejo en el uso del suelo.

\section{Referencias}

Álvarez de la Torre, G. (2010). El crecimiento urbano y estructura urbana en las Ciudades medias Mexicanas. Quivera Revista de Estudios Territoriales, 12(2), 94-115. Recuperado de: https:/ / quivera.uaemex.mx/article/view/10191

Balbo, M., Jordan, R., y Simioni, D. (2003). La Ciudad inclusiva. Santiago de Chile, Chile: CEPAL.

Cano, L., Rodríguez, R., Valdez, J., Acevedo, O. y Beltrán, R. (2016). Detección del crecimiento urbano en el estado de Hidalgo mediante imágenes Landsat. Investigaciones Geográficas. 92, 1-10. https://dx.doi.org/10.14350/rig.52339

Cifuentes, P. y Londoño, J. (2010). Análisis del crecimiento urbano: Una aproximación al estudio de los factores de crecimiento de la ciudad de Manizales como aporte a la planificación. Gestión y Ambiente 13(1), 53-66. Recuperado de https:/ / revistas.unal.edu.co/index.php/gestion/article/view/25384

CONAPO. (2005). Proyecciones de la población en México 2005-2030. Recuperado de: http:/ / www.conapo.gob.mx/index.php?option=com_content\&view=article\&id=36\&Itemid=234.

Delgado, M., Matteucci, S., Acevedo, M., Valeri, C., y J. Márquez, (2017). Causas directas que inducen el cambio de uso de suelo y de la cobertura boscosa, a escala de paisaje, en el sur de Venezuela. Interciencia, 42(3), 148-156. Recuperado de: http:/ / www.redalyc.org/articulo.oa?id=33950011002

García, C. (2010). “Movilidad Intermetropolitana en la región del Centro de México. El patrón del commuting en el México Central en el 2000" Ponencia presentada en la X Reunión Nacional de Investigación Demográfica en México, Escenarios Demográficos y Política de Población en el Siglo XXI, Sociedad Mexicana de Demografía. El Colegio de México, México D. F.

González, S. y Larralde, A. (2013). Conceptualización y medición de lo rural. Una propuesta para clasificar el espacio rural en México. En: CONAPO, Situación demográfica de México., México D.F: CONAPO. 141157.

Graizbord, B. (2002). Elementos para el ordenamiento territorial: uso del suelo y recursos. Estudios Demográficos y Urbanos, 17(2), 411-423. doi:http:/ / dx.doi.org/10.24201/edu.v17i2.1146

Granados, A. (2010). Los nuevos residentes de Pachuca. Pachuca, México: UAEH

INEGI. (2016). Conjunto de datos vectoriales de Uso del suelo y vegetación Serie VI. Escala 1:250,000. Obtenida a partir de la aplicación de técnicas de fotointerpretación con imágenes de satélite Landsat TM8 seleccionadas del año 2014. Aguascalientes, México: INEGI

INEGI. (2014a). Diccionario de datos de uso del suelo y vegetación. Escala 1:250, 000. Versión 3. México D.F.: INEGI

INEGI. (2014b). Características de las localidades 2014. Síntesis metodológica y conceptual. México D.F.: INEGI 


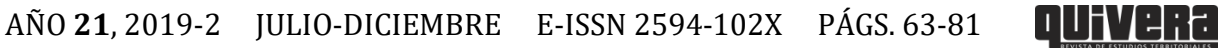

INEGI. (2013). Conjunto de datos vectoriales de uso de suelo y vegetación escala 1:250 000, serie V (capa unión), escala: 1:250000. Aguascalientes, México: INEGI

INEGI. (2010a). XIII Censo de población y vivienda. México D.F.: INEGI

INEGI. (2010b). Uso del suelo y vegetación. Escala Serie IV. 1:250,000. (continuo nacional). Aguascalientes, México: INEGI

INEGI. (2005a). Población rural y rural ampliada en México 2000. Aguascalientes, México: INEGI

INEGI (2005b). Uso del suelo y vegetación. Escala Serie III. 1:250,000 (continuo nacional). Aguascalientes, México: INEGI

INEGI. (2001). Uso del suelo y vegetación. Serie II. Escala 1:250,000 (continuo nacional). Aguascalientes, México: INEGI

INEGI. (2000). XII Censo de población y vivienda. México D.F.: INEGI

INEGI. (1990). XI Censo de población y vivienda. México D.F.: INEGI

INE e INEGI. (1997). Carta de Uso del suelo y vegetación. Serie I. Escala 1:250,000 (continuo nacional). Digitalización de las cartas de uso del suelo y vegetación elaboradas por INEGI entre los años 1980-1991 con base en fotografías aéreas de 1968-1986. México D.F.: INEGI

Lezama, J., y Domínguez, J. (2006). Medio ambiente y sustentabilidad urbana. Papeles de población, 12(49), 153176. Recuperado de: http://www.scielo.org.mx/scielo.php?script=sci_arttext\&pid=S140574252006000300007\&lng=es\&tlng=es.

López, V. H., Balderas, M. A., Chávez, M. C., Pérez, J. I., y Gutiérrez, J. G. (2015). Land Use Change and its Socio-Economic Implications in the Mazahua Area of the Mexican Highlands. CIENCIA Ergo-Sum, 22(2), 136-144. Recuperado de: https:/ / cienciaergosum.uaemex.mx/article/view/7714

Martínez, S. (2006). Lo urbano y lo rural, una relación indisociable: importancia del suelo de conservación del Distrito Federal. Revista Economía Informa. 339, 34-45. Recuperado de: http://www.economia.unam.mx/publicaciones/econinforma/pdfs/339/05sergiomartinez.pdf

Naciones Unidas. (2014). Naciones Unidas: Departamento de asuntos económicos y Sociales. Recuperado de: http://www.un.org/es/development/desa/news/population/world-urbanization-prospects2014.html

Pascual, V., Aguilera, F., Plata, W., Gómez, M. y Bosque, J. (2010). “Simulación de modelos de crecimiento urbano: métodos de comparación con los mapas reales”. En: Ojeda, J., Pita, M.F. y Vallejo, I. (Eds.), Tecnologías de la Información Geográfica: La Información Geográfica al servicio de los ciudadanos. Sevilla: Universidad de Sevilla..

Pérez, M. C, (2018). Expansión de la ciudad en la zona metropolitana de Pachuca: procesos desiguales y sujetos $\begin{array}{llll}\text { migrantes } & \text { e } & \text { Territorios, } & \text { 0(38), }\end{array}$ doi:http://dx.doi.org/10.12804/revistas.urosario.edu.co/territorios/a.5577

SEDATU, CONAPO, INEGI. (2018). Delimitación de las Zonas Metropolitanas de México 2015. México: SEDATU, CONAPO, INEGI. Recuperado de: https://www.gob.mx/conapo/documentos/delimitacion-de-laszonas-metropolitanas-de-mexico-2015

Tovar, E. (2011). Zonas metropolitanas en el estado de Hidalgo y cooperación intermunicipal. Argumentos 24(66), 155-179. Recuperado de: http://www.scielo.org.mx/scielo.php?script=sci_arttext\&pid=S0187$57952011000200007 \& \operatorname{lng}=$ es\&tlng=es

Villalvazo, P., Corona, J. P., y García, S. (2002). Urbano-rural, constante búsqueda de fronteras conceptuales. Revista de información y análisis, 20, 17-24. Recuperado de: http:/ / bibliotecadigital.imipens.org/uploads/Urbanorural, $\% 20$ constante $\% 20$ busqueda $\% 20$ de $\% 20$ fronteras $\% 20$ conceptuales $\% 20$-.pdf 